\title{
GLOBAL ESTIMATES FOR KERNELS OF NEUMANN SERIES AND GREEN'S FUNCTIONS
}

\author{
MICHAEL FRAZIER, FEDOR NAZAROV, AND IGOR E. VERBITSKY
}

ABSTRACT. We obtain global pointwise estimates for kernels of the resolvents $(I-T)^{-1}$ of integral operators

$$
T f(x)=\int_{\Omega} K(x, y) f(y) d \omega(y)
$$

on $L^{2}(\Omega, \omega)$ under the assumptions that $\|T\|_{L^{2}(\omega) \rightarrow L^{2}(\omega)}<1$ and $d(x, y)=1 / K(x, y)$ is a quasi-metric. Let $K_{1}=K$ and $K_{j}(x, y)=$ $\int_{\Omega} K_{j-1}(x, z) K(z, y) d \omega(z)$ for $j \geq 1$. Then

$$
K(x, y) e^{c K_{2}(x, y) / K(x, y)} \leq \sum_{j=1}^{\infty} K_{j}(x, y) \leq K(x, y) e^{C K_{2}(x, y) / K(x, y)}
$$

for some constants $c, C>0$.

Our estimates yield matching bilateral bounds for Green's functions of the fractional Schrödinger operators $(-\triangle)^{\alpha / 2}-q$ with arbitrary nonnegative potentials $q$ on $\mathbb{R}^{n}$ for $0<\alpha<n$, or on a bounded non-tangentially accessible domain $\Omega$ for $0<\alpha \leq 2$. In probabilistic language, these results can be reformulated as explicit bilateral bounds for the conditional gauge associated with Brownian motion or $\alpha$-stable Lévy processes.

2010 Mathematics Subject Classification. Primary 42B20, 42B37. Secondary $60 \mathrm{~J} 65,81 \mathrm{Q} 15$.

Key words and phrases. Neumann series, Green's function, quasi-metric kernels, Schrödinger operators, non-tangentially accessible domains, conditional gauge.

The second author is supported in part by NSF grant DMS-1249196. The third author is supported in part by NSF grant DMS-1161622. 


\section{Contents}

1. Introduction

2. Estimates for Kernels of Neumann Series

3. Further Results on Quasi-metric and Quasi-metrically Modifiable Kernels

\section{INTRODUCTION}

This paper is dedicated to bounds for kernels of resolvents $(I-T)^{-1}$ of integral operators

$$
T f(x)=\int_{\Omega} K(x, y) f(y) d \omega(y)
$$

and their applications to estimates for Green's functions of Schrödinger operators and related quantities. Throughout, $\omega$ is a positive measure on $\Omega$.

We consider the formal Neumann series

$$
(I-T)^{-1}=I+\sum_{j=1}^{\infty} T^{j}
$$

and the associated kernels $K_{1}=K$ and

$$
K_{j}(x, y)=\int_{\Omega} K_{j-1}(x, z) K(z, y) d \omega(z)
$$

for $j \geq 2$, of the operators $T^{j}$. Define the formal Green's function $H: \Omega \times \Omega \rightarrow(0,+\infty]$ by

$$
H(x, y)=\sum_{j=1}^{\infty} K_{j}(x, y) .
$$

Let $\|T\|=\|T\|_{L^{2}(\omega) \rightarrow L^{2}(\omega)}$ denote the operator norm of $T$ on $L^{2}(\omega)$.

We will consider the class of quasi-metric kernels, which have been considered previously in several papers, for example [KV] and [H]. A quasi-metric kernel $K$ on a measure space $(\Omega, \omega)$ is a measurable function from $\Omega \times \Omega$ into $(0, \infty]$ such that

(i) $K$ is symmetric: $K(x, y)=K(y, x)$ for all $x, y \in \Omega$, and

(ii) $d=1 / K$ satisfies the quasi-triangle inequality

$$
d(x, y) \leq \kappa(d(x, z)+d(z, y))
$$


for all $x, y, z \in \Omega$, for some $\kappa>0$, called the quasi-metric constant for $K$.

Our main theorem is the following.

Theorem 1.1. Let $(\Omega, \omega)$ be a $\sigma$-finite measure space. Let $K$ be a quasi-metric kernel on $\Omega$. Suppose $\|T\|<1$. Then there exists $c=$ $c(\kappa)>0$ and $C=C(\kappa,\|T\|)>0$ such that

$$
K(x, y) e^{c K_{2}(x, y) / K(x, y)} \leq H(x, y) \leq K(x, y) e^{C K_{2}(x, y) / K(x, y)} .
$$

It is well-known (see Lemma 2.1) that if $\|T\|>1$, then $H(x, y)=$ $+\infty$ for all $x$ and $y$. In the critical case $\|T\|=1$, the lower bound still holds, but there are examples where $H$ is finite a.e. and also examples where $H=+\infty$ a.e., although $K_{2}$ is finite a.e.

Kernels of the form $K(x, y)=\sum_{Q} c_{Q} \chi_{Q}(x) \chi_{Q}(y)$, where the sum is over all dyadic cubes in $\mathbb{R}^{n}$, were considered in [FV1], in connection with a discrete model of the Schrödinger equation (see Remark 2.8). Such kernels are quasi-metric with quasi-metric constant 1. Estimates of the form of inequality (1.4) were obtained in [FV1], under a Carleson condition on the sequence of scalars $\left\{c_{Q}\right\}$. (A sharp constant in the Carleson condition is established below; see Remark 2.8.)

In [FV2], estimate (1.4) and (1.7), (1.8) below were obtained under stronger assumptions.

Estimate (1.4) immediately extends (see Corollary 3.3) to the more general class of quasi-metrically modifiable kernels. A map $K: \Omega \times$ $\Omega \rightarrow(0, \infty]$ is quasi-metrically modifiable with constant $\kappa$ if there exists a measurable function $m: \Omega \rightarrow(0, \infty)$ such that $\widetilde{K}(x, y)=$ $K(x, y) /(m(x) m(y))$ is a quasi-metric kernel on $\Omega$ with quasi-metric constant $\kappa$. We call $m$ a modifier of $K$.

Our main application is to the fractional Schrödinger operator

$$
\mathcal{L}_{\alpha}=(-\triangle)^{\alpha / 2}-q
$$

with nonnegative potential $q \in L_{l o c}^{1}(\Omega)$ in some (possibly unbounded) domain $\Omega \subseteq \mathbb{R}^{n}$. Let $G(x, y)=G^{(\alpha)}(x, y)$ be the Green's kernel associated with the fractional Laplacian $(-\Delta)^{\alpha / 2}$ on $\Omega$ (see [L], BBK], [H] for references and definitions). We note that $G(x, y)$ is non-negative and symmetric on $\mathbb{R}^{n} \times \mathbb{R}^{n}$, and $G(x, y)=0$ if $x \in(\bar{\Omega})^{c}, y \in \mathbb{R}^{n}$. For regular domains $\Omega$, this is true if $x \in \Omega^{c}$. For the sake of simplicity we will assume throughout the paper that domains $\Omega$ are open and connected, so that $G(x, y)>0$ in $\Omega \times \Omega$, although most estimates remain true without the connectedness assumption. 
By $G f$ we denote the corresponding Green potential operator, that is,

$$
G f(x)=\int_{\Omega} G(x, y) f(y) d y, \quad x \in \Omega .
$$

For appropriate $f$ and $\Omega$, we have $G f=0$ in $\Omega^{c}$ and $(-\triangle)^{\alpha / 2} G f=f$ in $\Omega$. More generally,

$$
G \mu(x)=\int_{\Omega} G(x, y) d \mu(y), \quad x \in \Omega
$$

where $\mu$ is a Borel measure on $\Omega$.

Let $q$ be a non-negative, locally integrable function on $\Omega$. Let

$$
d \omega(x)=q(x) d x .
$$

Let $G_{1}=G$ and define $G_{j}$ inductively for $j \geq 2$ by

$$
G_{j}(x, y)=\int_{\Omega} G_{j-1}(x, z) G(z, y) d \omega(z) .
$$

The minimal Green's function associated with the fractional Schrödinger operator $\mathcal{L}_{\alpha}=(-\triangle)^{\alpha / 2}-q$ is

$$
\mathcal{G}(x, y)=\sum_{j=1}^{\infty} G_{j}(x, y) .
$$

The corresponding Green's operator is

$$
\mathcal{G} f(x)=\int_{\Omega} \mathcal{G}(x, y) f(y) d y .
$$

Formally, $u=\mathcal{G} f$ is the solution of the integral equation

$$
u(x)=\int_{\Omega} G(x, y) u(y) d \omega(y)+G f(x), \quad x \in \Omega, \quad \text { a.e. in } \Omega,
$$

and hence, by applying $G$, to the Schrödinger equation

$$
\mathcal{L}_{\alpha} u=(-\triangle)^{\alpha / 2} u-q u=f .
$$

Theorem 1.2 below, which yields estimates for $\mathcal{G}$ like those for $H$ in Theorem 1.1, is applicable in the following cases.

(1) If $\Omega=\mathbb{R}^{n}$ and $0<\alpha<n$, then $G$ is the classical Riesz kernel $G^{(\alpha)}(x, y)=c_{n, \alpha}|x-y|^{\alpha-n}$, which is a quasi-metric kernel.

(2) If $\Omega$ is a ball or half-space, then for all $0<\alpha<n, G=G^{(\alpha)}$ is a quasi-metrically modifiable kernel with modifier $m(x)=\delta(x)^{\alpha / 2}$, where $\delta(x)$ is the distance from $x$ to the boundary $\partial \Omega$. This is easy to see from the concrete form of Green's kernel in these cases. 
(3) If $\Omega$ is a bounded domain with $C^{1,1}$ boundary then

$$
G^{(\alpha)}(x, y) \approx \frac{\delta(x)^{\alpha / 2} \delta(y)^{\alpha / 2}}{|x-y|^{n-\alpha}(|x-y|+\delta(x)+\delta(y))^{\alpha / 2}},
$$

where " $\approx$ " means that the ratio of the two sides is bounded above and below by positive constants depending only on $\Omega$, holds for $0<\alpha \leq 2$, $\alpha<n$. Hence $G=G^{(\alpha)}$ is a quasi-metrically modifiable kernel with modifier $m(x)=\delta(x)^{\alpha / 2}$.

(4) If $\Omega$ is a bounded domain satisfying the boundary Harnark principle and $0<\alpha \leq 2$, then $G=G^{(\alpha)}$ is quasi-metrically modifiable with modifier $m(x)=\min \left(1, G\left(x, x_{0}\right)\right)$, for $x_{0} \in \Omega$, with a quasi-metric constant $\kappa$ independent of $x_{0}$. In particular, this procedure is applicable when $\Omega$ is a bounded Lipschitz domain, or more generally an NTA (non-tangentially accessible) domain. In fact, for $0<\alpha<2$, it suffices to assume that $\Omega$ is merely an interior NTA domain which obeys the interior corkscrew condition. This class of $\Omega$ coincides with the class of uniform (or $\kappa$-fat) domains. See [An], [BBK], [H], [K], [FV2], p. 118, for references and further discussion.

For the examples just listed, the following bilateral estimate follows immediately from the extension of Theorem 1.1 to the case of quasimetrically modifiable kernels. Our upper estimate is new even in the classical case $\alpha=2$; the lower estimate is known for $0<\alpha \leq 2$ in the cases (1)-(4) discussed above (see [GH], and the literature cited there).

Theorem 1.2. Let $\Omega \subseteq \mathbb{R}^{n}, n \geq 2$. Assume that the Green's kernel $G$ for $(-\Delta)^{\alpha / 2}$ on $\Omega$ is quasi-metrically modifiable. Let $q \in L_{l o c}^{1}(\Omega)$ be non-negative, and set $d \omega=q d x$. Define $\mathcal{G}$ by (1.5). Then there exists a positive constant $c=c(\Omega, \alpha)$ such that

$$
\mathcal{G}(x, y) \geq G(x, y) e^{c G_{2}(x, y) / G(x, y)} .
$$

If, in addition, $\|T\|<1$, where $T$ is the operator $T f(x)=\int_{\Omega} G(x, y) f(y) d \omega(y)$, then there exists a positive constant $C=$ $C(\Omega, \alpha,\|T\|)$ such that

$$
\mathcal{G}(x, y) \leq G(x, y) e^{C G_{2}(x, y) / G(x, y)} .
$$

When $\alpha=2$, there is a precise probabilistic formula

$$
\mathcal{G}(x, y) / G(x, y)=E_{x, y}\left[e^{\int_{0}^{\zeta} q\left(X_{t}\right) d t}\right],
$$

where $X_{t}$ is the Brownian path, with properly rescaled time, starting at $x$, and $E_{x, y}$ is the conditional expectation conditioned on the event that $X_{t}$ hits $y$ before exiting $\Omega$, and $\zeta$ is the time when $X_{t}$ first hits $y$. The expression $E_{x, y}\left[e^{\int_{0}^{\zeta} q\left(X_{t}\right) d t}\right]$ is called the conditional gauge, or the 
Feynman-Kac functional of the conditioned process (see [AS, CZ]). Recently, similar formulas have been established in the case $0<\alpha<2$ for the conditional gauge associated with an $\alpha$-stable Lévy process (see $[\mathrm{BBK}]$ ). However, our approach is more general and covers even some cases with $\alpha>2$ (in particular, $\Omega=\mathbb{R}^{n}$ ) for which there seems to be no probabilistic interpretation.

This probabilistic approach yields the lower bound (1.7) with $c=1$, by applying Jensen's inequality in (1.9). On the other hand, the upper estimate (1.8), which can be rewritten as

$$
E_{x, y}\left[e^{\int_{0}^{\zeta} q\left(X_{t}\right) d t}\right] \leq e^{C E_{x, y}\left[\int_{0}^{\zeta} q\left(X_{t}\right) d t\right]},
$$

seems to be new. It would be interesting to see if it has a probabilistic proof.

Some nonlinear analogues of Theorem 1.2 for quasilinear equations of the $p$-Laplace type with natural growth terms are obtained in [JV1, JV2. However, they are less precise and do not determine sharp constants in the conditions on $q$.

In Section 2, we prove Theorem 1.1. We discuss further results concerning integral operators with quasi-metric or quasi-metrically modifiable kernels in Section 3 .

\section{Estimates for Kernels of Neumann Series}

Lemma 2.1. Let $(\Omega, \omega)$ be a $\sigma$-finite measure space, and let $K: \Omega \times$ $\Omega \rightarrow(0, \infty]$ be a symmetric kernel on $\Omega$. Define $T$ by (1.1). Let $K_{1}=K$ and define $K_{j}$ by (1.2) for $j \geq 2$. If $\|T\|>1$, then for every $x, y \in \Omega$,

$$
H(x, y)=\sum_{j=1}^{\infty} K_{j}(x, y)=+\infty .
$$

Proof. Suppose there exist $x_{0}, y_{0} \in \Omega$ such that $H\left(x_{0}, y_{0}\right)<\infty$. Then

$$
\begin{gathered}
\int_{\Omega} H\left(x_{0}, z\right) K\left(z, y_{0}\right) d \omega(z)=\int_{\Omega} \sum_{j=1}^{\infty} K_{j}\left(x_{0}, z\right) K\left(z, y_{0}\right) d \omega(z) \\
=\sum_{j=1}^{\infty} K_{j+1}\left(x_{0}, y_{0}\right)<H\left(x_{0}, y_{0}\right)<\infty .
\end{gathered}
$$

Since $K\left(z, y_{0}\right)>0$ for all $z \in \Omega$, we see that $H\left(x_{0}, z\right)<\infty$ for a.e. $z$. 
Let $f(x)=H\left(x_{0}, x\right)$. Then by the symmetry of $H$,

$$
\begin{aligned}
T f(x)= & \int_{\Omega} K(x, z) H\left(z, x_{0}\right) d \omega(z)=\sum_{j=1}^{\infty} \int_{\Omega} K(x, z) K_{j}\left(z, x_{0}\right) d \omega(z) \\
& =\sum_{j=1}^{\infty} K_{j+1}\left(x, x_{0}\right)<H\left(x, x_{0}\right)=H\left(x_{0}, x\right)=f(x) .
\end{aligned}
$$

Since $0<f(x)<\infty$ a.e., Schur's Lemma implies that $\|T\| \leq 1$.

We turn to the proof of the lower estimate for $H$ in Theorem 1.1 . It is only meaningful for $\|T\| \leq 1$, by the previous lemma, but the proof does not involve $\|T\|$.

We say that $d$ is a quasi-metric with quasi-metric constant $\kappa$, on a nonempty set $\Omega$, if $d: \Omega \times \Omega \rightarrow[0, \infty)$ is not identically 0 and satisfies $d(x, y)=d(y, x)$ and the quasi-triangle inequality (1.3) for all $x, y, z \in \Omega$.

We will use the fact that $\kappa \geq 1 / 2$. To see this fact, select $x \in \Omega$. If $d(x, x)>0$, applying (1.3) with $x=y=z$ gives $\kappa \geq 1 / 2$. If $d(x, x)=0$, then there must exist $y \in \Omega$ such that $d(x, y)>0$, and applying (1.3) with $z=x$ implies that $\kappa \geq 1$. Note that $\kappa=1 / 2$ is attained in the case where $d$ is constant.

Lemma 2.2. (Ptolemy) Let $d$ be a quasi-metric with constant $\kappa$ on a set $\Omega$. Suppose $y_{1}, y_{2}, y_{3}, y_{4} \in \Omega$. Let $a=d\left(y_{1}, y_{2}\right), b=d\left(y_{2}, y_{3}\right), c=$ $d\left(y_{3}, y_{4}\right), d=d\left(y_{4}, y_{1}\right), s=d\left(y_{2}, y_{4}\right)$, and $t=d\left(y_{1}, y_{3}\right)$. Then

$$
s t \leq 4 \kappa^{2} \max (a c, b d) \text {. }
$$

Proof. Without loss of generality, assume $a=\min (a, b, c, d)$. Then

$$
s \leq \kappa(a+d) \leq 2 \kappa d \text { and } t \leq \kappa(a+b) \leq 2 \kappa b .
$$

Hence $s t \leq 4 \kappa^{2} b d$.

Lemma 2.3. Let $(\Omega, \omega)$ be a $\sigma$-finite measure space, and let $K$ be a quasi-metric kernel on $\Omega$. Let $K_{1}=K$ and define $K_{j}$ by (1.2) for $j \geq 2$. Then for $c=\left(4 \kappa^{2}\right)^{-1}$,

$$
\sum_{j=1}^{\infty} K_{j}(x, y) \geq K(x, y) e^{c K_{2}(x, y) / K(x, y)} .
$$

Proof. Let $d=1 / K$. We can assume $d(x, y)>0$ for all $x, y$. To see this, for $n \in \mathbb{N}$, let $K^{(n)}=\min (K, n)$. Then $K^{(n)}$ is a quasi-metric with the same quasi-metric constant as for $K$, corresponding to $d_{n}=$ $\max (d, 1 / n)$. Using (2.1) for $K^{(n)}$ yields the result for $K$. Note that 
at points where $K(x, y)=\infty$ or $K_{2}(x, y)=\infty$, both sides of (2.1) are infinite.

Fix $(x, y) \in \Omega$. For $z \in \Omega$, define

$$
F(z)=\frac{d(x, z)}{d(y, z)}
$$

For $j \geq 2$, let

$$
A_{j}=\left\{\left(z_{1}, \ldots, z_{j-1}\right) \in \Omega^{j-1}: F\left(z_{1}\right) \leq F\left(z_{2}\right) \leq \cdots \leq F\left(z_{j-1}\right)\right\} .
$$

For $z=\left(z_{1}, \ldots, z_{j-1}\right) \in A_{j}$, we have

$$
d\left(x, z_{m+1}\right) d\left(y, z_{m}\right) \geq d\left(x, z_{m}\right) d\left(y, z_{m+1}\right)
$$

for $m=1, \ldots, j-2$, and hence, by Lemma 2.2 ,

$$
d\left(z_{m}, z_{m+1}\right) d(x, y) \leq 4 \kappa^{2} d\left(x, z_{m+1}\right) d\left(y, z_{m}\right) .
$$

Therefore, letting $d \omega_{j-1}(z)=d \omega\left(z_{1}\right) d \omega\left(z_{2}\right) \cdots d \omega\left(z_{j-1}\right)$, we get

$$
\begin{gathered}
K_{j}(x, y)=\int_{\Omega^{j-1}} \frac{1}{d\left(x, z_{1}\right)} \frac{1}{d\left(z_{1}, z_{2}\right)} \cdots \frac{1}{d\left(z_{j-2}, z_{j-1}\right)} \frac{1}{d\left(z_{j-1}, y\right)} d \omega_{j-1}(z) \\
\geq\left(\frac{d(x, y)}{4 \kappa^{2}}\right)^{j-2} \int_{A_{j}} \frac{1}{d\left(x, z_{1}\right)} \frac{1}{d\left(z_{1}, y\right)} \frac{1}{d\left(x, z_{2}\right)} \frac{1}{d\left(z_{2}, y\right)} \cdots \\
\cdots \frac{1}{d\left(x, z_{j-1}\right)} \frac{1}{d\left(z_{j-1}, y\right)} d \omega_{j-1}(z)
\end{gathered}
$$

This last integral is invariant under permutations of the indices $1, \ldots, j-1$ in the definition of $A_{j}$, and hence has value at least $\frac{1}{(j-1) !}$ times the integral over all of $\Omega^{j-1}$, which splits and gives the value $K_{2}(x, y)^{j-1}$. Therefore

$$
K_{j}(x, y) \geq c^{-1} K(x, y) \frac{\left(c K_{2}(x, y) / K(x, y)\right)^{j-1}}{(j-1) !},
$$

with $c^{-1}=4 \kappa^{2} \geq 1$. We sum these estimates over $j \geq 2$ and add $K(x, y)=K_{1}(x, y)$ to obtain

$$
\sum_{j=1}^{\infty} K_{j}(x, y) \geq K(x, y) e^{c K_{2}(x, y) / K(x, y)} .
$$

Now we turn to the upper estimate of $H$ in Theorem 1.1. The following lemma is standard (see [Hei], Proposition 14.5), except for the value of the constants, which we will use. As indicated in the proof in [Hei] (pp. 111-112), the inequality below holds with $\beta \geq 2 \log _{2}(2 \kappa)$ and $C=(2 \kappa)^{2}$. Notice that in the proof in [Hei] the quasi-ultra-metric 
condition $d(x, y) \leq K \max [d(x, z), d(y, z)]$ is used in place of (1.3), so the constant $K$ should be replaced with $2 \kappa$.)

Lemma 2.4. [Hei] Let $d$ be a quasi-metric with constant $\kappa$ on set $\Omega$. Then there exists a quasi-metric D with constant 1 such that

$$
D^{\beta} \leq d \leq C D^{\beta}
$$

for $\beta=2 \log _{2}(2 \kappa)$ and $C=(2 \kappa)^{2}$.

Note that $D$ may not be a metric because we may have $D(x, x)>0$ or $D(x, y)=0$ for $x \neq y$. The proof in Hei] can easily be adapted to this case.

Lemma 2.5. (Inverse Ptolemy) Let $D$ be a quasi-metric with constant 1 on a set $\Omega$. Let $y_{1}, y_{2}, y_{3}, y_{4} \in \Omega$. Let $a=D\left(y_{1}, y_{2}\right), b=D\left(y_{2}, y_{3}\right), c=$ $D\left(y_{3}, y_{4}\right), d=D\left(y_{4}, y_{1}\right), s=D\left(y_{2}, y_{4}\right)$, and $t=D\left(y_{1}, y_{3}\right)$. Suppose the inequality

$$
a c \geq \tau^{2} b d
$$

holds with some $\tau>1$. Then

$$
s t \geq\left(1-\tau^{-1}\right)^{2} a c .
$$

Proof. Without loss of generality, $a \geq c$. Since $a^{2} \geq a c \geq \tau^{2} b d$, either $a \geq \tau b$ or $a \geq \tau d$. We can assume $a \geq \tau b$. Then

$$
t \geq a-b \geq\left(1-\tau^{-1}\right) a .
$$

Simce $a c \geq \tau^{2} b d$, either $a \geq \tau d$ or $c \geq \tau b$. In the first case,

$$
s \geq a-d \geq\left(1-\tau^{-1}\right) a \geq\left(1-\tau^{-1}\right) c .
$$

In the second case,

$$
s \geq c-b \geq\left(1-\tau^{-1}\right) c
$$

Hence we always have the estimate

$$
s t \geq\left(1-\tau^{-1}\right) a\left(1-\tau^{-1}\right) c=\left(1-\tau^{-1}\right)^{2} a c .
$$

Corollary 2.6. Let $d$ be a quasi-metric with constant $\kappa$ on a set $\Omega$, and let $D$ be the quasi-metric with constant 1 determined in Lemma 2.4. Let $x, y, u, v \in \Omega$. Suppose that for some $\tau>1$,

$$
\frac{D(x, v)}{D(y, v)} \geq \tau^{2} \frac{D(x, u)}{D(y, u)} \text {. }
$$

Then

$$
d(x, y) d(u, v) \geq\left(1-\tau^{-1}\right)^{2 \beta}(2 \kappa)^{-4} d(x, v) d(y, u) .
$$


Proof. The result can be immediately obtained by raising both sides of the estimate in Lemma 2.5 to the power $\beta$ and applying the bilateral inequality (2.2) for $d$ and $D^{\beta}$.

We now turn to the proof of the upper estimate in Theorem 1.1. Define the quasi-metric $d=1 / K$. Let $D$ be the quasi-metric with constant 1 determined in Lemma 2.4. By considering $\min (K, n)$ as in the proof of Lemma 2.3 and applying the monotone convergence theorem, we can assume $D(x, y)>0$ for all $x, y \in \Omega$. Fix $x, y \in \Omega$ and define the function $F$ by

$$
F(z)=\frac{D(x, z)}{D(y, z)}, \quad z \in \Omega
$$

and $f$ by

$$
f(z)=\frac{1}{\sqrt{d(x, z) d(y, z)}}, \quad z \in \Omega .
$$

Fix $\tau>1$ and $j \geq 2$. The heart of the proof is the following pointwise estimate.

Lemma 2.7. Let $\tau>1$, and let $F$ and $f$ be defined by (2.3) and (2.4) respectively. For every chain of points $z_{1}, z_{2}, \ldots, z_{j-1}$ in $\Omega$, there exists a subset

$$
M=\left\{m_{1}, m_{2}, \ldots, m_{\ell}\right\} \subseteq\{1, \ldots, j-2\},
$$

with cardinality $|M|$, with $m_{1}<m_{2}<\cdots<m_{\ell}$ such that

$$
F\left(z_{m_{k}}\right)<F\left(z_{m_{k+1}}\right) \text { for all } k=1,2, \ldots, \ell-1
$$

and

$$
A \leq(2 \kappa)^{2} C(\tau, \kappa)^{|M|} \tau^{\beta(j-2-|M|)} d(x, y)^{|M|} B
$$

where

$$
A=\frac{1}{d\left(x, z_{1}\right)} \frac{1}{d\left(z_{1}, z_{2}\right)} \cdots \frac{1}{d\left(z_{j-2}, z_{j-1}\right)} \frac{1}{d\left(z_{j-1}, y\right)}
$$

and

$$
\begin{aligned}
B & =f\left(z_{1}\right) \frac{1}{d\left(z_{1}, z_{2}\right)} \cdots \frac{1}{d\left(z_{m_{1}-1}, z_{m_{1}}\right)} f\left(z_{m_{1}}\right) \\
& \times \prod_{k=1}^{\ell-1}\left[f\left(z_{m_{k}+1}\right) \frac{1}{d\left(z_{m_{k}+1}, z_{m_{k}+2}\right)} \cdots \frac{1}{d\left(z_{m_{k+1}-1}, z_{m_{k+1}}\right)} f\left(z_{m_{k+1}}\right)\right] \\
& \times f\left(z_{m_{\ell}+1}\right) \frac{1}{d\left(z_{m_{\ell}+1}, z_{m_{\ell}+2}\right)} \cdots \frac{1}{d\left(z_{j-2}, z_{j-1}\right)} f\left(z_{j-1}\right) .
\end{aligned}
$$


Proof. For $m=1, \ldots, j-1$, define

$$
\Phi(m)=\min _{k \geq m} F\left(z_{k}\right) .
$$

Then $\Phi$ is nondecreasing. Let

$$
M=\left\{m \in\{1,2, \ldots, j-2\}: \Phi(m+1) \geq \tau^{2} \Phi(m)\right\} .
$$

Notice that $F\left(z_{m}\right) \geq \Phi(m)$ and $F\left(z_{m}\right)=\Phi(m)$ for $m \in M$. Hence $F\left(z_{m}\right)$ is increasing for $m \in M$, because $\Phi$ is increasing, so (2.5) holds.

For $m \in M$,

$$
F\left(z_{m+1}\right) \geq \Phi(m+1) \geq \tau^{2} F\left(z_{m}\right)
$$

We have

$$
\frac{A}{B}=\sqrt{\frac{d\left(y, z_{1}\right)}{d\left(x, z_{1}\right)}}\left[\prod_{m \in M} \frac{\sqrt{d\left(x, z_{m}\right) d\left(y, z_{m}\right) d\left(x, z_{m+1}\right) d\left(y, z_{m+1}\right)}}{d\left(z_{m}, z_{m+1}\right)}\right] \sqrt{\frac{d\left(x, z_{j-1}\right)}{d\left(y, z_{j-1}\right)}} .
$$

The estimate $F\left(z_{m+1}\right) \geq \tau^{2} F\left(z_{m}\right)$ means that the conditions of Corollary 2.6 hold for the points $x, y, z_{m}, z_{m+1}$ for every $m \in M$. Thus

$$
d(x, y) d\left(z_{m}, z_{m+1}\right) \geq\left(1-\tau^{-1}\right)^{2 \beta}(2 \kappa)^{-4} d\left(x, z_{m+1}\right) d\left(y, z_{m}\right) .
$$

Hence

$$
\begin{aligned}
\frac{A}{B} & \leq\left[\frac{(2 \kappa)^{4}}{\left(1-\tau^{-1}\right)^{2 \beta}}\right]^{|M|} d(x, y)^{|M|} \\
& \times \sqrt{\frac{d\left(y, z_{1}\right)}{d\left(x, z_{1}\right)}}\left[\prod_{m \in M} \sqrt{\frac{d\left(x, z_{m}\right) d\left(y, z_{m+1}\right)}{d\left(x, z_{m+1}\right) d\left(y, z_{m}\right)}}\right] \sqrt{\frac{d\left(x, z_{j-1}\right)}{d\left(y, z_{j-1}\right)}} .
\end{aligned}
$$

By the equivalence of $d$ and $D^{\beta}$, we can estimate the last quantity by

$$
\begin{aligned}
\frac{A}{B} & \leq\left[(2 \kappa)^{2}\right]^{|M|+1}\left[\frac{(2 \kappa)^{4}}{\left(1-\tau^{-1}\right)^{2 \beta}}\right]^{|M|} d(x, y)^{|M|} \\
& \times\left[\frac{D\left(y, z_{1}\right)}{D\left(x, z_{1}\right)}\left(\prod_{m \in M} \frac{D\left(x, z_{m}\right) D\left(y, z_{m+1}\right)}{D\left(x, z_{m+1}\right) D\left(y, z_{m}\right)}\right) \frac{D\left(x, z_{j-1}\right)}{D\left(y, z_{j-1}\right)}\right]^{\beta / 2} \\
& =(2 \kappa)^{2}\left[\frac{(2 \kappa)^{6}}{\left(1-\tau^{-1}\right)^{2 \beta}}\right]^{|M|} d(x, y)^{|M|}\left[\frac{1}{F\left(z_{1}\right)}\left(\prod_{m \in M} \frac{F\left(z_{m}\right)}{F\left(z_{m+1}\right)}\right) F\left(z_{j-1}\right)\right]^{\beta / 2}
\end{aligned}
$$

Note that $F\left(z_{1}\right) \geq \Phi(1), F\left(z_{j-1}\right)=\Phi(j-1)$, and recall that for every $m \in M$, we have $F\left(z_{m}\right)=\Phi(m)$ and $F\left(z_{m+1}\right) \geq \Phi(m+1)$. Hence we can estimate the product

$$
\frac{1}{F\left(z_{1}\right)}\left(\prod_{m \in M} \frac{F\left(z_{m}\right)}{F\left(z_{m+1}\right)}\right) F\left(z_{j-1}\right)
$$


by

$$
\frac{1}{\Phi(1)}\left(\prod_{m \in M} \frac{\Phi(m)}{\Phi(m+1)}\right) \Phi(j-1)=\frac{\Phi\left(m_{1}\right)}{\Phi(1)} \frac{\Phi\left(m_{2}\right)}{\Phi\left(m_{1}+1\right)} \cdots \frac{\Phi(j-1)}{\Phi\left(m_{\ell}+1\right)} .
$$

We now observe that the inequality $\Phi(m+1) \leq \tau^{2} \Phi(m)$ holds for every $m \in\left[1, m_{1}-1\right] \cup\left[m_{1}+1, m_{2}-1\right] \cup \cdots \cup\left[m_{\ell}+1, j-1\right]$, i.e., for $m \notin M$, so $\Phi\left(m_{1}\right) \leq \tau^{2\left(m_{1}-1\right)} \Phi(1), \Phi\left(m_{2}\right) \leq \tau^{2\left(m_{2}-m_{1}-1\right)} \Phi\left(m_{1}+1\right)$, and so on up to $\Phi(j-1) \leq \tau^{2\left(j-1-m_{\ell}-1\right)} \Phi\left(m_{\ell}+1\right)$. Therefore the last product does not exceed $\tau^{2(j-2-|M|)}$. Combining these estimates we get the conclusion of the lemma with $C(\tau, \kappa)=(2 \kappa)^{6}\left(1-\tau^{-1}\right)^{-2 \beta}$.

Proof of Theorem 1.1. Let $F$ and $f$ be defined by (2.3) and (2.4) respectively. Integrating the estimate (2.6) with respect to $\omega_{j-1}$ and summing over all possible choices of $M$, we arrive at the inequality

$$
\begin{aligned}
K_{j}(x, y) & \leq(2 \kappa)^{2} \sum_{\ell=0}^{j-2} C(\tau, \kappa)^{\ell} \tau^{\beta(j-1-\ell)} d(x, y)^{\ell} \\
& \times \sum_{1 \leq m_{1}<m_{2}<\cdots<m_{\ell} \leq j-2} I_{j}\left(m_{1}, m_{2}, \cdots, m_{\ell}\right),
\end{aligned}
$$

for $j \geq 2$, where

$$
\begin{aligned}
& I_{j}\left(m_{1}, m_{2}, \cdots, m_{\ell}\right) \\
& =\int_{\left\{F\left(z_{m_{1}}\right)<F\left(z_{m_{2}}\right)<\cdots<F\left(z_{m_{\ell}}\right)\right\}} \\
& \left(f T^{m_{1}-1} f\right)\left(z_{m_{1}}\right)\left(\prod_{k=1}^{\ell-1}\left(f T^{m_{k+1}-m_{k}-1} f\right)\left(z_{m_{k+1}}\right)\right)\left(f T^{j-1-m_{\ell}-1} f\right)\left(z_{j-1}\right) \\
& d \omega\left(z_{m_{1}}\right) \cdots d \omega\left(z_{m_{\ell}}\right) d \omega\left(z_{j-1}\right) .
\end{aligned}
$$

Let $\alpha \in\left(1,\|T\|^{-1}\right)$. Define $S=\sum_{k \geq 0} \alpha^{k} T^{k}$. Since $f \geq 0$, we have the pointwise inequality

$$
T^{k} f \leq \alpha^{-k} S f
$$

for all $k \geq 0$. Thus

$$
\begin{aligned}
& I_{j}\left(m_{1}, \ldots m_{\ell}\right) \leq \alpha^{-(j-2-\ell)} \int_{\left\{F\left(z_{m_{1}}\right)<F\left(z_{m_{2}}\right)<\cdots<F\left(z_{m_{\ell}}\right)\right\}} \\
& g\left(z_{m_{1}}\right) \cdots g\left(z_{m_{\ell}}\right) g\left(z_{j-1}\right) d \omega\left(z_{m_{1}}\right) \cdots d \omega\left(z_{m_{\ell}}\right) d \omega\left(z_{j-1}\right) \\
& \leq \frac{\alpha^{-(j-2-\ell)}}{\ell !}\|g\|_{L^{1}(\omega)}^{\ell+1},
\end{aligned}
$$


where $g=f S f$. The last inequality is true because the integral is invariant with respect to the permutations of $m_{1}, \ldots, m_{\ell}$ in the domain of integration.

Note that

$$
\|g\|_{L^{1}(\omega)} \leq\|S\|\|f\|_{L^{2}(\omega)}^{2}=\|S\| K_{2}(x, y) .
$$

Putting these estimates together, we obtain

$$
K_{j}(x, y) \leq(2 \kappa)^{2} K_{2}(x, y) \sum_{\ell=0}^{j-2}\left(\begin{array}{c}
j-2 \\
\ell
\end{array}\right)\left(\tau^{\beta} \alpha^{-1}\right)^{j-2-\ell} \frac{C(\tau, \kappa)^{\ell}}{\ell !}\left(\frac{K_{2}(x, y)}{K(x, y)}\right)^{\ell} .
$$

If $0<\rho<1$, then for each $\ell \geq 0$,

$$
\sum_{j=\ell}^{\infty}\left(\begin{array}{l}
j \\
\ell
\end{array}\right) \rho^{j-\ell}=\frac{1}{(1-\rho)^{\ell+1}}
$$

by differentiating $(1-\rho)^{-1}=\sum_{j=0}^{\infty} \rho^{j}$ a total of $\ell$ times. Select $\tau>1$ such that $\rho \equiv \tau^{\beta} \alpha^{-1}<1$. Using (2.7),

$$
\sum_{j=2}^{\infty} K_{j}(x, y) \leq \frac{(2 \kappa)^{2}}{1-\tau^{\beta} \alpha^{-1}} K_{2}(x, y) \exp \left(\frac{C(\tau, \kappa)}{1-\tau^{\beta} \alpha^{-1}} \frac{K_{2}(x, y)}{K(x, y)}\right) .
$$

To complete the proof of the upper bound in (1.4), it remains only to use the elementary inequality $1+C V e^{C V} \leq e^{2 C V}$, valid for all $C, V>0$.

Remark 2.8. Theorem 1.1 is applicable to the discrete model of the Schrödinger equation considered in [FV1]. Let $\omega$ be a Borel measure on $\mathbb{R}^{n}$, and let $\mathcal{Q}$ denote the family of dyadic cubes in $\mathbb{R}^{n}$. For a sequence $s=\left\{s_{Q}\right\}_{Q \in \mathcal{Q}}$ of positive scalars, we consider an operator $T$ defined by (1.1) with kernel

$$
K(x, y)=\sum_{Q \in \mathcal{Q}} \frac{s_{Q}}{\omega(Q)} \chi_{Q}(x) \chi_{Q}(y)
$$

where the sum is taken over all dyadic cubes $Q$ such that $\omega(Q) \neq 0$. This is a quasi-metric kernel with constant $\kappa=1$ (moreover, $d(x, y)=$ $1 / K(x, y)$ is an ultra-metric; that is, $d(x, y) \leq \max (d(x, z), d(z, y)))$. Note that, for $g \in L^{2}(\omega)$,

$$
\langle T g, g\rangle=\sum_{Q \in \mathcal{Q}} \frac{s_{Q}}{\omega(Q)}\left(\int_{Q} g d \omega\right)^{2} \leq\|T\| \cdot\|g\|_{L^{2}(\omega)}^{2} .
$$


Define the discrete Carleson norm of $s=\left\{s_{Q}\right\}_{Q \in \mathcal{Q}}$ by

$$
\|s\|_{\omega}=\sup _{Q \in \mathcal{Q}} \omega(Q)^{-1} \sum_{P \in \mathcal{Q}: P \subseteq Q} s_{P} \omega(P) .
$$

Then $\|s\|_{\omega} \leq\|T\| \leq 4\|s\|_{\omega}$, where the constant 4 is sharp (see [NTV], Theorem 3.3). Consequently, by Theorem 1.1 estimate (1.4) holds if $\|s\|_{\omega}<\frac{1}{4}$, where the constant $\frac{1}{4}$ is sharp as well. Indeed, (1.4) yields $\|T\| \leq 1$ by Schur's lemma, and so $\frac{1}{4}$ cannot be replaced by any larger constant in view of Theorem 3.3 in [NTV].

Such estimates of the corresponding Green's function were obtained earlier in [FV1] by a different method, with $\frac{1}{12}$ in place of $\frac{1}{4}$, along with estimates of solutions to the discrete Schrödinger equation $u=T u+f$.

\section{Further Results on QuASI-METRIC AND QuASI-METRICALly MODIFIABLE KERNELS}

Let $T$ be defined by (1.1) where $K: \Omega \times \Omega \rightarrow(0,+\infty]$ is a nonnegative kernel. The minimal positive solution $u_{0}$ of the equation $u=$ $T u+1$ is obviously given by $u_{0}=1+\sum_{j=1}^{\infty} T^{j} 1$. Our next result is a bilateral pointwise estimate of $u_{0}$. In the case where $K$ is the Green's function $G$ of $(-\Delta)^{\alpha / 2}$, the function $u_{0}=\mathcal{G} 1$ is of interest in the study of Schrödinger equations.

Theorem 3.1. Let $(\Omega, \omega)$ be a $\sigma$-finite measure space. Suppose that $K$ is a quasi-metric kernel with constant $\kappa$ on $(\Omega, \omega)$, and $T$ is the corresponding integral operator. Then there exists $c=c(\kappa)>0$ such that the minimal positive solution $u_{0}$ of the equation $u=T u+1$ satisfies

$$
u_{0} \geq e^{c T 1} \text {. }
$$

If $\|T\|<1$, then there exists $C=C(\kappa,\|T\|)>0$ such that

$$
u_{0} \leq e^{C T 1} \text {. }
$$

Proof. We first consider the case where $\Omega$ is bounded with respect to $d=1 / K$, that is, when $D=\sup _{x, y \in \Omega} d(x, y)<+\infty$. We will add a point $z$ to $\Omega$ which is far away from all other points. That is, we choose $z \notin \Omega$ and consider the space $\Omega^{*}=\Omega \cup\{z\}$ with quasi-metric $d^{*}$ defined by $d^{*}(x, y)=d(x, y)$ if $x, y \in \Omega, d^{*}(x, z)=d^{*}(z, x)=D$ for all $x \in \Omega$, and $d(z, z)=0$. Then $d^{*}$ is a quasi-metric on $\Omega^{*}$ with quasi-metric constant $\kappa^{*}=\max (\kappa, 1)$. We also extend $\omega$ to a measure $\omega^{*}$ on $\Omega^{*}$ by setting $\left.\omega^{*}\right|_{\Omega}=\omega$, and $\omega^{*}(\{z\})=0$.

Note that the iterates $K_{j}^{*}$ of $K^{*}=1 / d^{*}$ with respect to $\omega^{*}$ agree with the iterates $K_{j}$ of $K$ with respect to $\omega$ on $\Omega \times \Omega$ since $\omega^{*}(\{z\})=0$, 
and that the norm of the integral operator with the kernel $K^{*}$ on $\left(\Omega^{*}, \omega^{*}\right)$ is the same as $\|T\|$.

For all $x \in \Omega$,

$$
\frac{K_{2}^{*}(x, z)}{K^{*}(x, z)}=D \int_{\Omega^{*}} K^{*}(x, y) K^{*}(y, z) d \omega^{*}(y)=\int_{\Omega} K(x, y) d \omega(y)=T 1(x)
$$

and

$$
\begin{gathered}
u_{0}(x)=1+\sum_{j=1}^{\infty} \int_{\Omega} K_{j}(x, y) d \omega(y)=1+D \sum_{j=1}^{\infty} \int_{\Omega^{*}} K_{j}^{*}(x, y) K^{*}(y, z) d \omega^{*}(y) \\
=D K^{*}(x, z)+D \sum_{j=2}^{\infty} K_{j}^{*}(x, z)=D \sum_{j=1}^{\infty} K_{j}^{*}(x, z) .
\end{gathered}
$$

Hence, applying the lower estimate in Theorem 1.1 on the space $\Omega^{*}$ we get, for all $x \in \Omega$,

$$
u_{0}(x) \geq D K^{*}(x, z) e^{c K_{2}^{*}(x, z) / K^{*}(x, z)}=e^{c T 1(x)} .
$$

Similarly, the upper estimate in Theorem 1.1 gives, for all $x \in \Omega$,

$$
u_{0}(x) \leq D K^{*}(x, z) e^{C K_{2}^{*}(x, z) / K^{*}(x, z)}=e^{C T 1(x)} .
$$

For $\Omega$ not bounded with respect to $d$, select $x_{0} \in \Omega$ and let $\Omega_{n}=$ $\left\{x \in \Omega: d\left(x, x_{0}\right)<n\right\}$. Let $\omega_{n}$ be the restriction of $\omega$ to $\Omega_{n}$, and let $d_{n}$ and $K^{(n)}$ be the restrictions of $d$ and $K$ to $\Omega_{n} \times \Omega_{n}$ respectively. Then $K^{(n)}$ is a quasi-metric kernel on $\Omega_{n}$. The corresponding integral operator $T_{n}$ defined by

$$
T_{n} f(x)=\int_{\Omega_{n}} K^{(n)}(x, y) f(y) d \omega_{n}(y)=\int_{\Omega} K(x, y) \chi_{\Omega_{n}}(y) d \omega(y)
$$

satisfies

$$
\left\|T_{n}\right\|_{L^{2}\left(\Omega_{n}\right) \rightarrow L^{2}\left(\Omega_{n}\right)} \leq\|T\|_{L^{2}(\Omega) \rightarrow L^{2}(\Omega)},
$$

and $T_{n} 1 \rightarrow T 1$ pointwise as $n \rightarrow \infty$.

Let $T_{n}^{j}$ be the $j^{\text {th }}$ iterate of $T_{n}$ and let $K_{j}^{(n)}$ be the kernel of $T_{n}^{j}$. Then $K_{j}^{(n)}(x, y)$ is non-decreasing in $n$ and converges to $K_{j}(x, y)$ pointwise as $n \rightarrow \infty$, for each $j \in \mathbb{N}$, by the monotone convergence theorem. Let $u_{0}^{(n)}=1+\sum_{j=1}^{\infty} T_{n}^{j} 1$. By the monotone convergence theorem, $u_{0}^{(n)} \rightarrow u_{0}$ pointwise as $n \rightarrow \infty$.

Applying the estimates for the bounded space $\Omega_{n}$, and passing to the limit as $n \rightarrow \infty$, we see that estimates (3.1) and (3.2) hold in the unbounded case as well.

We now turn to characterizing the kernels for which $\sum_{j=1}^{\infty} K_{j}(x, y)$ is pointwise equivalent to $K(x, y)$. 
Theorem 3.2. Let $(\Omega, \omega)$ be a $\sigma$-finite measure space. Suppose $K$ : $\Omega \times \Omega \rightarrow(0,+\infty]$ is a quasi-metric kernel with constant $\kappa$, and that $K$ is not identically $\infty$. Let $T$ be the integral operator corresponding to $K$ and let $u_{0}$ be the minimal positive solution of the equation $u=T u+1$. Then the following statements are equivalent:

(a) There exists $C_{1}>0$ such that $\sum_{j=1}^{\infty} K_{j}(x, y) \leq C_{1} K(x, y)$ for all $x, y \in \Omega$.

(b) $\|T\|<1$ and $K_{2}(x, y) \leq C_{2} K(x, y)$ for all $x, y \in \Omega$, for some $C_{2}>0$ (or, equivalently, $\sup _{x \in \Omega} T 1(x)<+\infty$ ).

(c) $\sup _{x \in \Omega} u_{0}(x)<+\infty$.

Proof. We first show that the condition $K_{2} \leq C_{2} K$ is equivalent to the boundedness of $T 1$. Notice that by the quasi-metric property of $K$,

$$
K(x, z) K(y, z) \leq \kappa K(x, y)[K(x, z)+K(y, z)]
$$

Hence,

$$
\begin{aligned}
K_{2}(x, y) & =\int_{\Omega} K(x, z) K(y, z) d \omega(z) \\
& \leq \kappa K(x, y) \int_{\Omega} K(x, z) d \omega(z)+\kappa K(x, y) \int_{\Omega} K(y, z) d \omega(z) \\
& =\kappa K(x, y)[T 1(x)+T 1(y)]
\end{aligned}
$$

so that the boundedness of $T 1$ implies that $K_{2} \leq C_{2} K$.

Now suppose $K_{2} \leq C_{2} K$. Fix $x \in \Omega$. Suppose first that

(1) $0<\sup _{y \in \Omega} d(x, y)=D<+\infty$. Pick any $y \in \Omega$ with $d(x, y)>$ $\frac{D}{2}$. Then

$$
d(y, z) \leq \kappa[d(x, y)+d(x, z)] \leq 3 \kappa d(x, y)
$$

for all $z \in \Omega$, and so $K(y, z) \geq \frac{1}{3 \kappa} K(x, y)$. It follows that

$$
K_{2}(x, y)=\int_{\Omega} K(x, z) K(y, z) d \omega(z) \geq \frac{1}{3 \kappa} K(x, y) T 1(x),
$$

whence $T 1(x) \leq 3 \kappa C_{2}$.

Now suppose that

(2) $\sup _{y \in \Omega} d(x, y)=+\infty$. Then there is a sequence $y_{n} \in \Omega$ such that $0<r_{n}=d\left(x, y_{n}\right) \rightarrow+\infty$ as $n \rightarrow \infty$. For every $z \in B\left(x, r_{n}\right)$, we have

$$
d\left(y_{n}, z\right) \leq \kappa\left[d\left(x, y_{n}\right)+d(x, z)\right] \leq 2 \kappa d\left(x, y_{n}\right)
$$


Hence,

$$
\begin{aligned}
K_{2}\left(x, y_{n}\right) & \geq \int_{B\left(x, r_{n}\right)} K(x, z) K\left(y_{n}, z\right) d \omega(z) \\
& \geq \frac{1}{2 \kappa} K\left(x, y_{n}\right) \int_{B\left(x, r_{n}\right)} K(x, z) d \omega(z),
\end{aligned}
$$

and consequently $\int_{B\left(x, r_{n}\right)} K(x, z) d \omega(z) \leq 2 \kappa C_{2}$ for all $n \in \mathbb{N}$. Passing to the limit as $n \rightarrow \infty$, we get $T 1(x) \leq 2 \kappa C_{2}$.

Now Theorem 1.1 shows that (b) implies (a), and Theorem 3.1 shows that (b) implies (c).

If $u_{0}$ is bounded by $C$, then by Theorem 3.1, $T 1$ is bounded. From $u_{0}=T u_{0}+1$, we obtain

$$
T u_{0}=u_{0}-1 \leq\left(1-\frac{1}{C}\right) u_{0} .
$$

Hence $\|T\| \leq 1-1 / C<1$, by Schur's Lemma. So (c) implies (b).

It remains to show that (a) implies (b). Condition (a) trivially implies $K_{2} \leq C_{2} K$, and hence we have that $T 1$ is bounded. It remains to show that (a) implies $\|T\|<1$. Since the kernel of $T$ is positive, $T$ is bounded on $L^{\infty}(\omega)$, and by duality on $L^{1}(\omega)$. Thus by interpolation $T$ is a bounded operator on $L^{2}(\omega)$. Comparing kernels and using (a), there exists $C$ so that for all $n$,

$$
\left\|T^{n}\right\| \leq\left\|T+T^{2}+\cdots+T^{n}\right\| \leq C .
$$

Since the kernel of $T$ is symmetric, $T$ is self-adjoint, so $\|T\|$ coincides with the spectral radius $r(T)$ on $L^{2}(\omega)$. Hence it suffices to show that $\left\|T^{n}\right\|<1$ for some $n$. If not, given $n$ we can select $f$ such that $\|f\|=1$ and $\left\|T^{n} f\right\|>1 / 2$. We can assume $f \geq 0$. Then for all $m \leq n$

$$
\left\|T^{m} f\right\| \geq \frac{1}{2 C}
$$

Then

$$
\left\|\left(T+T^{2}+\cdots+T^{n}\right) f\right\|^{2} \geq \sum_{j=1}^{n}\left\|T^{j} f\right\|^{2} \geq \frac{n}{4 C^{2}},
$$

since all inner products in the expansion of the left side are nonnegative. For $n$ large enough, this inequality contradicts $\| T+T^{2}+$ $\cdots+T^{n} \| \leq C$. 
Remarks. 1. The condition $\sup _{x \in \Omega} T 1(x)<+\infty$ can be expressed in a "geometric form"

$$
\sup _{x \in \Omega} \int_{0}^{+\infty} \frac{\omega(B(x, t))}{t^{2}} d t<+\infty
$$

where $B(x, t)=\{y \in \Omega: d(x, y)<t\}$. Indeed,

$$
\begin{gathered}
T 1(x)=\int_{\Omega} K(x, y) d \omega(y)=\int_{\Omega} \frac{d \omega(y)}{d(x, y)} \\
=\int_{\Omega} \int_{d(x, y)}^{\infty} \frac{d t d \omega(y)}{t^{2}}=\int_{0}^{+\infty} \frac{\omega(B(x, t))}{t^{2}} d t .
\end{gathered}
$$

2. The condition $\sup _{x \in \Omega} T 1(x)<+\infty$ can be replaced with $\|T 1\|_{L^{\infty}(\omega)}<+\infty$, which in its turn is equivalent to $\|T\|_{L^{1}(\omega) \rightarrow L^{1}(\omega)}<$ $+\infty$.

Indeed, let $E=\left\{x: T 1(x) \leq\|T 1\|_{L^{\infty}(\omega)}\right\}$. Then $\omega(\Omega \backslash E)=0$, so $E$ is non-empty. Fix any point $x \in \Omega$. Then the following two cases are possible: (i) $\inf _{y \in E} d(x, y)=0$. In this case, for every $\epsilon>0$, there exists $y \in E$ such that $d(x, y)<\epsilon$, and therefore $d(y, z) \leq \kappa(d(x, z)+\epsilon)$, whence

$$
\int_{\Omega} \frac{d \omega(z)}{d(x, z)+\epsilon} \leq \kappa T 1(y) \leq \kappa\|T 1\|_{L^{\infty}(\omega)} .
$$

Passing to the limit as $\epsilon \rightarrow 0$, we obtain $T 1(x) \leq \kappa\|T 1\|_{L^{\infty}(\omega)}$.

(ii) $D=\inf _{y \in E} d(x, y)>0$. Then choose any point $y \in E$ with $d(x, y) \leq 2 D$. Note that for all $z \in E$,

$$
d(y, z) \leq \kappa(d(x, y)+d(x, z)) \leq \kappa(2 D+d(x, z)) \leq 3 \kappa d(x, z) .
$$

Thus in this case

$$
T 1(x)=\int_{E} \frac{d \omega(z)}{d(x, z)} \leq 3 \kappa \int_{E} \frac{d \omega(z)}{d(y, z)}=3 \kappa T 1(y) \leq 3 \kappa\|T 1\|_{L^{\infty}(\omega)} .
$$

3. Let $\mathcal{B}$ denote the space of all bounded functions on $\Omega$ with norm $\|u\|_{\mathcal{B}}=\sup _{x \in \Omega}|u(x)|$. Suppose $T$ is an integral operator with quasi-metric kernel. Clearly $T: \mathcal{B} \rightarrow \mathcal{B}$ is bounded if and only if $\sup _{x \in \Omega} T 1(x)<+\infty$. Under this additional assumption, W. Hansen [H] showed that condition (a) of Theorem 3.2 is equivalent to $r(T)_{\mathcal{B}}<1$, where $r(T)_{\mathcal{B}}$ is the spectral radius of $T$ in $\mathcal{B}$. This result is a consequence of Theorem 3.2 above. Moreover, for operators $T$ with quasimetric kernels which are bounded on $\mathcal{B}$, we have $r(T)_{\mathcal{B}}=\|T\|$.

Indeed, $\|T\|_{\mathcal{B} \rightarrow \mathcal{B}} \geq\|T\|_{L^{\infty}(\omega) \rightarrow L^{\infty}(\omega)}=\|T\|_{L^{1}(\omega) \rightarrow L^{1}(\omega)}$. Using interpolation, and the formula $r(T)_{\mathcal{B}}=\lim _{n \rightarrow \infty}\left\|T^{n}\right\|_{\mathcal{B} \rightarrow \mathcal{B}}^{1 / n}$ we see that $r(T)_{\mathcal{B}} \geq r(T)_{L^{2}(\omega)}=\|T\|$. By an argument similar to that used in the 
proof of Theorem 3.2 (with $\mathcal{B}$, or $L^{\infty}(\omega)$, in place of $L^{2}(\omega)$ ) it follows that (a) implies $r(T)_{\mathcal{B}}<1$. Thus, the condition $r(T)_{\mathcal{B}}<1$ is equivalent to $\|T\|<1$ for operators $T$ with quasi-metric kernels bounded on $\mathcal{B}$. To prove that $r(T)_{\mathcal{B}}=\|T\|$, it remains to notice that, for $\epsilon>0$, the operator $T_{\epsilon}=(\|T\|+\epsilon)^{-1} T$ satisfies $\left\|T_{\epsilon}\right\|<1$, and hence $r\left(T_{\epsilon}\right)_{\mathcal{B}}<1$, which yields $r(T)_{\mathcal{B}}<\|T\|+\epsilon$. Conversely, $S_{\epsilon}=\left(r(T)_{\mathcal{B}}+\epsilon\right)^{-1} T$ satisfies $r\left(S_{\epsilon}\right)_{\mathcal{B}}<1$ which gives $\left\|S_{\epsilon}\right\|<1$, that is, $\|T\|<r(T)_{\mathcal{B}}+\epsilon$. Letting $\epsilon \rightarrow 0$ yields $r(T)_{\mathcal{B}}=\|T\|$.

We will now extend our results to a wider class of quasi-metrically modifiable kernels. It turns out that in many interesting applications the quasi-metric property fails but the quasi-metric modifiability holds. Let $K$ be a quasi-metrically modifiable kernel on a measure space $(\Omega, \omega)$, with modifier $m$, so that $\widetilde{K}(x, y)=K(x, y) /(m(x) m(y))$ is a quasi-metric kernel.

We also consider the measure $d \widetilde{\omega}=m^{2} d \omega$ and the operator $\tilde{T}$ defined by

$$
\widetilde{T} f(x)=\int_{\Omega} \widetilde{K}(x, y) f(y) d \tilde{\omega}(y) .
$$

Various properties of a quasi-metrically modifiable kernel $K$ and the corresponding integral operator $T$ can be reduced to those of $\widetilde{K}$ and $\widetilde{T}$. The following properties are straightforward, and we leave their proofs to the reader.

(a) $\widetilde{K}_{j}(x, y)=\frac{K_{j}(x, y)}{m(x) m(y)}$ for all $j \in \mathbb{N}$.

(b) If $f \in L^{2}(\omega)$, then $\widetilde{f}=\frac{f}{m} \in L^{2}(\widetilde{\omega})$.

(c) $\widetilde{T}^{j} \tilde{f}=\frac{T^{j} f}{m}$, for all $j \in \mathbb{N}$.

(d) $\widetilde{T}^{j} 1=\frac{T^{j} m}{m}$, for all $j \in \mathbb{N}$.

(e) $\|\widetilde{T}\|_{L^{2}(\widetilde{\omega})}=\|T\|_{L^{2}(\omega)}$.

Applying Theorem 1.1 to $\widetilde{K}$ and $\widetilde{T}$, and rewriting the conclusions in terms of $K$ and $T$, we deduce that Theorem 1.1 remains valid verbatim for quasi-metrically modifiable kernels, which we state as the following corollary.

Corollary 3.3. Let $(\Omega, \omega)$ be a $\sigma$-finite measure space, and let $K$ be a quasi-metrically modifiable kernel on $\Omega$ with constant $\kappa$. Let $K_{1}=K$ 
and define $K_{j}$ by (1.2) for $j \geq 2$. Then there exists $c>0$, depending only on $\kappa$, such that (2.1) holds.

Define $T$ by (1.1). If $\|T\|_{L^{2}(\omega) \rightarrow L^{2}(\omega)}<1$, then there exists $C>0$, depending only on $\kappa$ and $\|T\|$, such that

$$
\sum_{j=1}^{\infty} K_{j}(x, y) \leq K(x, y) e^{C K_{2}(x, y) / K(x, y)} .
$$

Theorem 3.1 becomes a statement concerning the minimal positive solution $u_{0}$, defined by $u_{0}=m+\sum_{j=1}^{\infty} T^{j} m$, of the equation $u=$ $T u+m$, where $m$ is the quasi-metric modifier. We obtain estimates for $u_{0}$ in the next corollary which is deduced by applying Theorem 3.1 to $u_{0} / m=1+\sum_{j=1}^{\infty} \widetilde{T}^{j} 1$.

Corollary 3.4. Suppose $K$ is a quasi-metrically modifiable kernel with modifier $m$ and constant $\kappa$ on $(\Omega, \omega)$. Define $T$ by (1.1). Then there exists $c>0$ depending only on $\kappa$ such that

$$
u_{0} \geq m e^{c(T m) / m} \text {. }
$$

If $\|T\|<1$, then there exists $C>0$ depending only on $\kappa$ and $\|T\|$ such that

$$
u_{0} \leq m e^{C(T m) / m} .
$$

Moreover, $u_{0} \approx m$ if and only if $\|T\|<1$, and $T m \leq C m$.

The next corollary is a direct analogue of Theorem 3.2 for quasimetrically modifiable kernels, proved by reducing to the quasi-metric case via (a) - (e) above.

Corollary 3.5. Let $(\Omega, \omega)$ be a $\sigma$-finite measure space. Suppose $K$ : $\Omega \times \Omega \rightarrow(0,+\infty]$ is quasi-metrically modifiable with constant $\kappa$ and modifier $m$, and that $K$ is not identically $\infty$. Let $T$ be the integral operator corresponding to $K$ and let $u_{0}$ be the minimal positive solution of the equation $u=T u+m$. Then the following statements are equivalent:

(a) There exists $C_{1}>0$ such that $\sum_{j=1}^{\infty} K_{j}(x, y) \leq C_{1} K(x, y)$ for all $x, y \in \Omega$.

(b) $\|T\|<1$ and $K_{2}(x, y) \leq C_{2} K(x, y)$ for all $x, y \in \Omega$, for some $C_{2}>0$ (or, equivalently, $\sup _{x \in \Omega}(\operatorname{Tm}(x)) / m(x)<+\infty$ ).

(c) $\sup _{x \in \Omega}\left(u_{0}(x) / m(x)\right)<+\infty$.

In conclusion of this section we discuss an intrinsic characterization of the class of quasi-metrically modifiable kernels. Recall that a positive 
symmetric kernel $K$ is quasi-metrically modifiable with modifier $m>0$ if and only if $d(x, y)=m(x) m(y) D(x, y)$, where $D(x, y)=1 / K(x, y)$, is a quasi-metric. Then $D$ satisfies the Ptolemy inequality:

$$
D\left(y_{1}, y_{3}\right) D\left(y_{2}, y_{4}\right) \leq 4 \kappa^{2}\left(D\left(y_{1}, y_{2}\right) D\left(y_{3}, y_{4}\right)+D\left(y_{1}, y_{4}\right) D\left(y_{2}, y_{3}\right)\right)
$$

for all $y_{1}, y_{2}, y_{3}, y_{4} \in \Omega$. Indeed, by Lemma 2.2. we have such an inequality for $d$. Using the relation between $d$ and $D$ and cancelling the term $m\left(y_{1}\right) m\left(y_{2}\right) m\left(y_{3}\right) m\left(y_{4}\right)$ yields the Ptolemy inequality for $D$.

On the other hand, suppose $K$ is a postive, symmetric kernel with the property that for some $w \in \Omega, K(x, w)<\infty$ for all $x$, such that $D=1 / K$ satisfies the Ptolemy inequality

$$
D(x, y) D(z, w) \leq C(D(x, z) D(y, w)+D(x, w) D(y, z))
$$

for all $x, y, z \in \Omega$. Then dividing by $D(x, w) D(y, w) D(z, w)$ yields

$$
\frac{D(x, y)}{D(x, w) D(y, w)} \leq C\left(\frac{D(x, z)}{D(x, w) D(z, w)}+\frac{D(y, z)}{D(y, w) D(z, w)}\right) .
$$

Hence $m(x)=1 / D(x, w)=K(x, w)$ is a quasi-metric modifier for $K$. More generally, if $\omega(\{x: K(x, w)=\infty\})=0$, then $\{x: K(x, w)=\infty\}$ can be deleted from $\Omega$ without significant effect, and the contrary case is somewhat degenerate. Such observations about quasi-metric modifiers were first noticed by Hansen and Netuka [HN] (Proposition 8.1).

\section{REFERENCES}

[AS] M. Aizenman And B. Simon, Brownian motion and Harnack inequality for Schrödinger operators, Comm. Pure Appl. Math. 35 (1982), 209-273.

[An] A. AnCOnA, Some results and examples about the behaviour of harmonic functions and Green's functions with respect to second order elliptic operators, Nagoya Math. J. 165 (2002), 123-158.

[BBK] K. Bogdan, T. Byczkowski, T. Kulczycki, M. Ryznar, R. Song, And Z. VondraceK, Potential Analysis of Stable Processes and its Extensions, Lecture Notes Math. 1980, Springer-Verlag, Berlin, 2009.

[CZ] K. L. Chung and Z. ZhaO, From Brownian Motion to Schrödinger's Equation, Grundlehren der math. Wissenschaften 312, Springer, BerinHeidelberg, 1995.

[FV1] M. Frazier And I. E. Verbitsky, Solvability conditions for a discrete model of Schrödinger's equation, Analysis, Partial Differential Equations and Applications, Operator Theory: Adv. Appl. 193 (2009) Birkhäuser, Basel, 65-80.

[FV2] M. Frazier And I. E. Verbitsky, Global Green's function estimates, Around the Research of Vladimir Maz'ya III, Analysis and Applications, Ari Laptev, ed., International Mathematics Series 13 (2010), 105-152.

[GH] A. GRIGOR'YAN AND W. HANSEn, Lower estimates for a perturbed Green function, J. d'Anal. Math. 104 (2008), 25-58. 
[H] W. HANSEN, Uniform boundary Harnack principle and generalized triangle property, J. Funct. Anal. 226 (2005), 452-484.

[HN] W. Hansen and I. Netuka, On the Picard principle for $\Delta+\mu$, Math. Z. 270 (2012), 783-807.

[Hei] J. Heinonen, Lectures on Analysis on Metric Spaces, Universitext, Springer-Verlag, New York, 2001.

[JV1] B. J. JAYE AND I. E. VERBITSKY, The fundamental solution of nonlinear operators with natural growth terms, Ann. Sc. Norm. Super. Pisa Cl. Sci. (5) 12 (2013), 93-139.

[JV2] B. J. JAYE AND I. E. VERBITSKY, Local and global behaviour of solutions to nonlinear equations with natural growth terms, Arch. Rational Mech. Anal. 204 (2012), 627-681.

[KV] N. J. KAlton AND I. E. Verbitsky, Nonlinear equations and weighted norm inequalities, Trans. Amer. Math. Soc. 351 (1999), 3441-3497.

[K] C. E. KenIG, Harmonic Analysis Techniques for Second Order Elliptic Boundary Value Problems, CBMS Regional Conf. Series in Math. 83, Amer. Math. Soc., 1994.

[L] N. S. LANDKof, Foundations of Modern Potential Theory, Grundlehren der math. Wissenschaften, 180, Springer-Verlag, New York-Heidelberg, 1972.

[NTV] F. Nazarov, S. Treil, And A. Volberg, Bellman function in stochastic control and harmonic analysis, Systems, approximation, singular integral operators, and related topics, Oper. Theory Adv. Appl. 129 (2001) Birkhäuser, Basel, 393-423.

Mathematics Department, University of Tennessee, Knoxville, Tennessee 37922

E-mail address: frazier@math.utk.edu

Mathematics Department, Kent State University, Kent, Ohio 44242

E-mail address: nazarov@math.kent.edu

Department of Mathematics, University of Missouri, Columbia, Missouri 65211

E-mail address: verbitskyi@missouri.edu 